\title{
The process of pregnancy smoking cessation: implications for interventions
}

\author{
Carlo C DiClemente, Patricia Dolan-Mullen, Richard A Windsor
}

University of

Maryland Baltimore

County, Baltimore,

Maryland, USA

C C DiClemente

Centre for Health

Promotion Research and Prevention,

University of

Texas-Houston Health

Science Center, School

of Public Health,

Houston, Texas, USA

P Dolan-Mullen

University of Alabama, Birmingham,

Alabama, USA

R A Windsor

Correspondence to:

Carlo C DiClemente, $\mathrm{PhD}$

Psychology Department,

University of Maryland

Baltimore County, 1000

Hilltop Circle, Baltimore,

MD 21250, USA;

diclemen@umbc.edu

Received 20 January 2000

and in revised for

28 March 2000

Accepted 5 April 2000

\begin{abstract}
Objective-There is a growing body of knowledge about the pregnant smoker and what happens as she goes through the pregnancy and postpartum periods. This article reviews the process of smoking cessation in the context of pregnancy.

Data sources-Epidemiological data, extant reviews of the literature, and current original research reports are used to examine characteristics of the women and of the change process for those women smokers who quit, stop, or modify their smoking during pregnancy and the postpartum period.

Data synthesis-An analysis of the interaction of the process of smoking cessation with pregnancy was conducted to gain insight into the unique problems faced by the pregnant smoker and discover directions for intervention.

Conclusions-Pregnancy and the postpartum period provide a window of opportunity to promote smoking cessation and smoke free families. Understanding obstacles and pathways for pregnancy and postpartum smoking cessation can guide implementation of effective existing programs and development of new ones. Recommendations include promoting cessation before and at the beginning of pregnancy, increasing delivery of treatment early in pregnancy, helping spontaneous and intervention assisted quitters to remain tobacco free postpartum, aiding late pregnancy smokers, and involving the partner of the woman smoker.

(Tobacco Control 2000;9(Suppl III):iii16-iii21)
\end{abstract}

Keywords: pregnancy; smoking; process of change; relapse

Over the past 40 years findings from epidemiological research have demonstrated the serious consequences of cigarette smoking during pregnancy on the health and well being of the

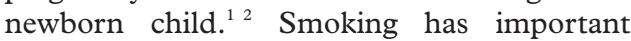
deleterious effects on the baby at birth and throughout the early development of the child. Deleterious effects are exacerbated if the mother and/or father continue to smoke during the early postnatal period. The good news is that over the past 20 years numerous clinical trials have demonstrated the effectiveness of smoking cessation interventions early in the pregnancy. ${ }^{3-12}$ Other studies have highlighted the cost effectiveness of these interventions in terms of birth outcomes. ${ }^{13}$ Although efficacy is not as well established, interventions in paediatric settings that target new mothers who continue or relapse to smoking during the postpartum period have begun to demonstrate modest success. ${ }^{15}$ Finally, intervention studies are beginning to target partners who smoke and to include fathers as well as the mothers in family interventions. ${ }^{16}{ }^{17}$ Studies of smoking cessation in the context of the pregnancy and birth of a child have yielded important information about the course and process of smoking cessation during the pregnancy and postpartum periods. This review outlines conceptual and practical considerations surrounding smoking cessation in the context of the birth of a child into the family. The objective is to review recent literature and highlight what is known about smoking cessation and pregnancy to promote effective strategies that can create smoke free families.

\section{Smoking during pregnancy}

The pregnant smoker comes from the larger population of female smokers of childbearing age. Demographic characteristics of this population provide important information about where the problem of pregnancy smoking is most likely to occur. Prevalence estimates indicate that $23 \%$ of the total population of women and approximately $25-30 \%$ of younger women (ages $15-30$ years) smoke cigarettes. $^{18}$ However, smoking is concentrated among white women in the lower socioeconomic strata (SES) of society who are less educated. ${ }^{18}{ }^{19}$ For African American, Hispanic, and Asian populations of women cigarette smoking is generally less prevalent across all age ranges with few exceptions. ${ }^{18}$

Rates of initiation are high and rates of cessation lowest among low SES, white women for a number of reasons. ${ }^{192021}$ Younger smokers are often less worried about the long term health effects of smoking and are more vulnerable to the image advertising of the tobacco industry. Most advertising targeting women smokers focuses on themes of independence featuring young women as smokers. $^{22}$ Secondly, women appear to find some unique benefits of smoking (weight management, affect management) that make smoking part of their coping with life and increase dependence on nicotine. A third factor is that younger women have multiple demands and stressors related to having to perform competing roles of mother, homemaker, and employee. ${ }^{21}$ Finally, these women often are influenced by support systems that encourage nicotine use. ${ }^{23}$ Younger women who decide to 
smoke in the context of the current anti-smoking climate and social norms constitute a subgroup of the population that would be more resistant to cessation pressures and needs to be better understood. Interventions to reach these women even before they become pregnant represent the most effective early intervention for preventing smoking during pregnancy. However, these interventions will have to be potent and multifaceted.

\section{Pregnancy quitters}

Women smokers follow a number of different paths in dealing with their smoking, as they become pregnant. Many women who consciously plan to become pregnant are likely to consider the option of stopping smoking as they prepare to become pregnant. Although the number of women who quit smoking before becoming pregnant is not known, there must be a number of women who stop smoking before becoming pregnant or while trying to become pregnant. Self reported rates of smoking on entry to obstetrics care are generally lower than population rates and recently were estimated at $13.6 \% .{ }^{24}$ Although these are self reported rates and subject to some deception, it is clear that some women do quit smoking before the first prenatal visit. Typically these prepregnancy quitters are identified as non-smokers or ex-smokers when they arrive at the first prenatal visit. Since they have stopped smoking weeks or months before the initial clinic visit, they are considered non-smokers and are not given any type of intervention related to smoking during pregnancy. Although we do not know much about this group of women, they are probably very conscious of health risks of smoking both for themselves and their baby. Most are probably dedicated to becoming smoke free not only for the pregnancy but for life. Prepregnancy quitters typically sustain cessation throughout the pregnancy and postpartum period. However, depending on the strength of the smoking habit and the extent of their motivation to quit, these women could be vulnerable to a return to smoking, particularly if there were some stressful event or a shift in environmental support.

\section{Spontaneous quitters}

Another group of women smokers quit as soon as they learn that they are pregnant. These "spontaneous quitters", as they have been called, are highly motivated to protect their babies. They quit primarily for the health and welfare of the baby and only secondarily for themselves. Estimates of the number of these women vary by SES and educational attainment. ${ }^{24}$ There are dramatic demographic differences between the spontaneous quitters and the continuing pregnant women smokers. Race, educational level, income, and employment status are primary characteristics on which the women who stop smoking "spontaneously" differ from those who continue..$^{25} 26$ Less educated, lower SES, white, unemployed women who are more dependent on nicotine have the greatest risk of continued smoking during pregnancy. The strength of nicotine addiction measured by the years of smoking and cotinine concentrations is a major predictor of cessation versus continued smoking. ${ }^{27}$ Finally, partner smoking represents another important dimension that influences whether the woman will be a spontaneous quitter. ${ }^{28}{ }^{29}$ We need more detailed information about the spontaneous quitters and their motivations and skills that enable them to stop smoking for extended periods of time. Spontaneous quitting or stopping smoking for the pregnancy early in the pregnancy is an important phenomenon that offers significant protection for the fetus and should be promoted as much as possible. Thus, it would be important to reach women of child bearing age before or as they become pregnant with warnings and messages of risk and benefit in order to plant the seeds of quitting smoking for the pregnancy.

Most spontaneous quitters, estimated at $80-85 \%$, are able to maintain cessation of smoking throughout the pregnancy. ${ }^{25}{ }^{30}$ However, they relapse back to smoking in the postpartum period at rates that are astounding considering the extended period of abstinence from cigarettes that they have achieved. Over $70 \%$ of these spontaneous quitters go back to smoking by six months after the birth of the baby and this rate may be higher among the lower SES patients who were able to stop smoking for the pregnancy. ${ }^{31}{ }^{32}$ Interestingly, data on process of change variables indicate that many of these women are not quitting smoking but only stopping smoking for the duration of the pregnancy. ${ }^{33}$ Compared to nonpregnant women who are in the process of quitting smoking, spontaneous quitters look more like non-pregnant women smokers who are in contemplation or preparation stages of change before quitting rather than nonpregnant women in action for smoking cessation. ${ }^{33}{ }^{34}$ These women who stop smoking for the pregnancy seem to have suspended their smoking rather than to be truly quitting smoking. This lack of normal cessation coping activities may explain the large relapse rate in the first six months of the postpartum period despite the fact that these women have been off cigarettes for many months. ${ }^{3} 3031$

The challenge for preventing postpartum return to smoking for these spontaneous quitters appears to be to shift their motivation from baby to self and to increase specific cognitive and behavioural process activity during the last trimester of the pregnancy and in the transition between pregnancy and the postpartum period. One recent study found that by asking spontaneous quitters while they are pregnant about their postpartum goals and their efficacy to remain abstinent postpartum, researchers were able to predict the return to smoking after the birth of the baby. ${ }^{35}$ However, most smoking cessation efforts during pregnancy focus on the pregnant woman who continues to smoke. Although there are some recent interventions designed to prevent relapse, ${ }^{52}$ typically little is done to assist the women who quit for the pregnancy to prevent a 
return to smoking either during the pregnancy or after the birth of the baby. ${ }^{36}{ }^{37}$

\section{Pregnant women smokers}

Women who continue to smoke during pregnancy pose a different challenge. The differences in socioeconomic levels that are evident in the population of women smokers of child bearing age become more exaggerated among the group of pregnant women who continue to smoke. The largest numbers of continuing smokers who are pregnant come from the subgroup of lower SES women smokers. Among this group of pregnant smokers there are often multiple and complex problems in addition to nicotine addiction. ${ }^{19} 2132$ Pregnant women who continue to smoke tend to have more psychological and emotional problems, less support and financial resources, more family problems, and less residential stability. ${ }^{26}{ }^{27}$ While smoking for these women is often a stress reducer, pregnancy is most often a stress producer. This interaction is problematic for the woman and the baby. Thus, women who continue to smoke throughout the pregnancy typically have more psychosocial problems and more smoke filled home environments. Reaching these women who continue to smoke with sustained interventions that would be effective and set in the context of the woman's real life problems is a significant challenge for investigators and clinicians alike.

Although difficult, it is possible to get women who continue to smoke even after they discover that they are pregnant to quit. ${ }^{312}$ Interventions early in the pregnancy $(<20$ to 24 weeks) have been able to help some women to quit and thus to increase the pool of early pregnancy quitters. Ershoff and colleagues at Kaiser Permanente of Southern California ${ }^{4}$ and Windsor and colleagues in Alabama ${ }^{11} 131427$ have demonstrated the effectiveness of brief, well executed interventions using medical advice and self help materials. However, once pregnant women arrive at their third trimester continuing to smoke, it is very difficult for them to quit and they are no longer susceptible to minimal interventions. In the Birmingham trial II study none of the women recruited between 24 and 32 weeks of gestation quit or significantly reduced their smoking. ${ }^{13}$ It may be that only the more dependent, heavier smokers continue to smoke and that the lighter smokers are able to stop earlier in the pregnancy. ${ }^{27}$

It is not clear what type of intervention could assist last trimester smokers. Some have suggested that nicotine replacement in the first or second trimester could be added to possible interventions for the more nicotine dependent smokers. ${ }^{38}$ In a recent study investigators examined an intervention consisting of motivational interviewing strategies delivered over the telephone and enhanced with personalised feedback for continuing resistant smokers called "One To One". ${ }^{39}$ Cotinine outcomes at the end of pregnancy were promising for the women who received the entire dose of two telephone calls and a personalised feedback letter. However, it was difficult to deliver that dose to these women smokers, particularly in the last trimester of pregnancy.

\section{Harm reduction}

Pregnant women who continue to smoke throughout the pregnancy often reduce the number of cigarettes that they smoke. ${ }^{40}{ }^{41}$ The data indicate that many can reduce nicotine concentrations significantly $(\geqslant 50 \%)$ and appear to be able to sustain the reductions. Reduction of smoking during pregnancy offers some measure of protection for the child. ${ }^{40}$ Although reducing would never give as much protection as complete cessation, for the fetus less exposure to the effects of smoking in utero certainly is better than greater exposure. It is not clear whether it would be possible to get continuing smokers to stop completely during the final days of the pregnancy so that the actual delivery would be uncomplicated by the ingestion of smoke, nicotine, carbon monoxide, and smoking related chemicals. However, harm reduction strategies - such as stopping smoking for brief periods of time either in the pregnancy or at the delivery, significantly reducing the number of cigarettes smoked, and engaging in other health protection behaviours including taking vitamins and exercise ${ }^{42}$ should be considered for those women who continue to smoke during the last trimester. ${ }^{12}$

\section{Postpartum cessation and relapse}

During the postpartum period there are several important transitions that we are beginning to identify and understand. There are a few women who stop smoking during the postpartum period among the women who continued to smoke during pregnancy. ${ }^{15}$ The numbers are small. Postpartum cessation is most probably related to heightened awareness of environmental tobacco smoke's effect on the small baby. However, during this same postpartum period most of the spontaneous quitters and intervention assisted quitters return to smoking. The number of women who relapse during the postpartum period far exceeds the number of continuing pregnancy smokers who quit during this period. ${ }^{36}$ Although there is evidence that increasing numbers of pregnant women are stopping or reducing smoking to protect the baby, the overall permanent reduction in smoking among women as a result of pregnancy leaves much to be desired because of postpartum relapse.

There are many interventions that have targeted quitting during pregnancy and some that target the postpartum period and the paediatric care system. However, few interventions focus on the critical transition from pregnancy to the postpartum period marked by the birth of the baby. One recent randomised, controlled trial, Project PANDA, developed an intervention that mailed videos and newsletters to the women and their partners timed to arrive at intervals during the final weeks of the pregnancy and the first six weeks postpartum. ${ }^{43}$ The goal of this intervention was to prevent the transition back to smoking at the time when the spontaneous quitters were most vulnerable. Results from Project PANDA indicated signifi- 
cantly greater abstinence over the entire follow up period and at the 12 month follow up for the intervention group participants $(55 \%$ v $45 \%$ ) supporting the hypothesis that it is possible to decrease the return to smoking for some of these women. Women in the experimental condition were significantly more likely to be abstinent at almost every follow up time point compared to a standard care control group.

The period immediately after the birth of the child is a particularly difficult one for the mother. The return to smoking appears to be facilitated by stress, lack of sleep, concerns about weight loss, and the ability to protect the baby from smoke in other ways. Interestingly, the return to smoking seems postponed until after the baby is weaned for mothers who breastfeed the baby. Although the early postpartum period is a difficult time, the potential exists to be able to assist women who stopped during the pregnancy to move to permanent cessation. ${ }^{5} 314344$

\section{Partner smoking}

There is probably no single facilitator of smoking and the return to smoking for women that is more influential than having a partner who smokes. ${ }^{17} 293132$ Partner smoking appears to make a critical contribution both to the woman's continuing smoking during pregnancy as well as in the return to smoking postpartum for spontaneous quitters. The Project PANDA intervention described above included an intervention component that attempted to address partner smoking by sending to the identified partner a set of video and print materials similar to those sent to the women but tailored to the male perspective on pregnancy. An initial evaluation of these materials based on interviews with the women in the intervention condition indicated greater cessation among the men. Men appeared to read and use the materials sent to them and the intervention appeared to make a small but significant difference in smoking with $28 \%$ of the men compared to $14 \%$ of controls not smoking at three months postpartum, but no differences at six and 12 month follow ups. ${ }^{16}$ Involving partners in protecting the baby from exposure to tobacco smoke during pregnancy and postpartum periods is an area that needs continuing development of innovative interventions and evaluation research. ${ }^{16}{ }^{172844}$

\section{Recommendations}

Reducing the personal and financial costs of smoking in families and particularly during the period of pregnancy and the birth of a child are important societal goals. The challenges are to eliminate pregnancy smoking and fetal exposure, to protect the newborn and developing child, to preserve the health and well being of the parents, and to create truly smoke free families. What are the critical approaches to interventions needed to meet these challenges? The following recommendations recognise that the course of smoking and smoking cessation for mothers and fathers from prepregnancy to postpartum offers multiple points of interven- tion that require multiple and multicomponent types of intervention strategies.

1. PROMOTING CESSATION AMONG WOMEN CONSIDERING CHILDBEARING

Programs and providers should promote smoking cessation among women who are considering childbearing by shifting the emphasis on cessation before or as close to the beginning of pregnancy as possible. This suggestion is consistent with recommendations for preconception counselling recommended a decade ago by an expert panel on the content of prenatal care. ${ }^{45}$ Cessation before pregnancy offers the best protection for the infant and maximises the possibility that the woman would integrate intrinsic motives about quitting for her own health with the motivation to quit for the baby's health. Intrinsic motivation may prove more effective for sustained behaviour change. ${ }^{46}$ Smoking cessation interventions included as part of family planning programs, in the distribution of various methods of birth control, and added to pregnancy testing done at home or in the clinic office would represent some potential opportunities to reach women smokers and their partners before pregnancy. In addition, if we can incorporate messages that focus on the health of both mother and baby, we may be able to create the type of quitting that will be more durable postpartum. Certainly this intervention point may work best for the population of women who plan to get pregnant. The planners, however, in all likelihood would be the women who are more likely to quit later in the pregnancy anyway.

\section{REACHING THE PREGNANT SMOKER AS SOON AS} POSSIBLE

Programs should also be developed that reach the pregnant smoker as early as possible in the pregnancy and that follow her throughout the pregnancy to promote and support sustained smoking cessation for the remainder of the pregnancy. Women who have the most difficulty stopping smoking often experience multiple problems and lack resources. Providing access to obstetric care as early as possible and embedding smoking cessation interventions in a comprehensive approach to these women's problems would appear to have the greatest chance of success. However, more intervention is not necessarily better. ${ }^{47}$

There are three critical issues that need to be addressed when creating pregnancy smoking cessation programs. The first is that identification of smoking risk among pregnant women must use a broad definition of smoking risk that includes women who quit before becoming pregnant. However, if women feel that identification will bring harassment, they will be reluctant to self identify and interventions will not be able to reach and influence those in earlier stages of change and most in need. ${ }^{34} 4849$ Sensitivity and tact are needed when addressing the smoking issue with these women. The second important point derived from our better understanding of cessation during pregnancy is that, although 
many of the spontaneous quitters sustain cessation throughout pregnancy, some need additional assistance. Sensitive probes and offers of support throughout the pregnancy and not simply at the first prenatal visit could be helpful to these women. The final critical issue has to do with creating and sustaining intervention delivery systems that would ensure reliable, early, recurrent, and effective delivery of interventions throughout the pregnancy to all pregnant women. This would include training doctors and staff in obstetric and gynaecology clinics in effective methods for addressing smoking with pregnant women. Educating them about the process of change and the windows of opportunity for intervening with the pregnant woman and her partner should be considered minimal preparation. Teaching motivational and behavioural strategies to address each woman's concerns would also be needed. Creating office and clinic systems that institutionalise identification and intervention protocols would be critical. This is an ambitious agenda. However, the payoff is significant in terms of quality of life and health care costs. ${ }^{50}$ There are a number of research and clinical programs that have demonstrated efficacy and can be used as models for creating intervention programs for pregnant women in both public and private settings. ${ }^{49}$

3. MAINTAINING POSTPARTUM CESSATION

Programs should create interventions for women who are able to stop smoking for the duration of the pregnancy that shift the focus to the maintenance of postpartum cessation. These interventions should be initiated sometime toward the end of the pregnancy and reach into the early postpartum period. The focus should be on the continuation of cessation from smoking after the birth of the baby. For women who stop smoking for the baby during the prepregnancy and pregnancy periods the motivation and coping activities for change needs to shift from simply protecting the baby to protecting the woman's health. The focus of smoking cessation should broaden to include the well being of the woman and the goal of creating a smoke free family. ${ }^{32} 3349$

4. DEVELOPING DIFFERENT INTERVENTIONS FOR THE CONTINUING SMOKER

A different set of interventions should be developed for those women who continue to smoke during the later stages of the pregnancy. Intervention efforts should promote various behaviours like smoking reduction, abstinence during critical periods immediately before the birthing process, increasing other health protection behaviours like vitamins and exercise, and an emphasis on postpartum cessation. Many women who continue to smoke are concerned about the health of the baby but find stopping smoking very difficult to achieve. Some have had prior births with no or few serious consequences from smoking. Others are overwhelmed by emotional, financial, and family problems. Continuing to work with these women to achieve the possible rather than the optimal can contribute to the overall goals of protecting the baby and promoting cessation. Harm reduction strategies are appropriate here and can facilitate movement toward the optimal goals. ${ }^{12} 2021$

5. FOCUSING ON THE PREGNANT SMOKER'S PARTNER

Programs should begin to focus on one of the most ignored targets of intervention for pregnancy smoking cessation-the partner of the woman smoker. Whether he smokes or not, the partner is, in almost all cases, an important part of the pregnancy. However, the partner who smokes has been identified in multiple studies as a risk factor for smoking during pregnancy and for postpartum relapse. ${ }^{29}$ Few interventions include the partner. Interventions should find ways to involve the partner to create the smoke free family because he can be an important ally. Moreover, pregnancy may be a very opportune time to intervene with smoking and other health habits of the partner, including smoking. Parenthood brings with it a re-evaluation of lifestyle and family needs that offers an opportunity that should not be missed to engage the paternal member of the family in a discussion of values and health behaviours.

\section{Conclusion}

Pregnancy offers multiple windows of opportunity for smoking cessation intervention. The course of pregnancy and the reality of the postpartum period create the moving target for cessation efforts. The goal of creating smoke free families, however, cannot be achieved with a single, simple intervention. Understanding the process of change for smoking during pregnancy can give ideas and guidance about what is needed in our research efforts and clinical interventions to promote this important behaviour change. The above recommendations are based on our growing knowledge about smoking and pregnancy. We have learned a great deal about cigarette smoking during the course of pregnancy and the postpartum experience of the woman smoker. We are beginning to disseminate the most effective programs and to study and evaluate new approaches to achieving sustained cessation of smoking throughout the pregnancy and postpartum periods. As we achieve an even better understanding of the types of interventions and the appropriate timing of these interventions that would yield the best outcomes, we can create an array of interventions and a family of strategies that will be more effective, sensitive, and comprehensive.

1 US Department of Health and Human Services. The health consequences of smoking for women. A report of the Surgeon General, 1980. Rockville, Maryland: Office of the Assistant Secretary for Health, Office on Smoking and Health, 1980. (DHHS Publication No (CDC) 90-8416.)

2 Floyd, RL, Rimer, BK, Giovino, GA, Mullen, PD, Sullivan, SE. A review of smoking in pregnancy: Effects on
pregnancy outcomes and cessation efforts. Annu Rev Public Health 1993;14:379-411.

3 Dolan-Mullen P, Ramirez G, Groff J. A meta-analysis of randomized trials of prenatal smoking cessation interventions. Am f Obstet Gynecol 1994;171:1328-34. 
4 Ershoff DH, Mullen PD, Quinn VP. A randomized trial of a serialized self-help smoking cessation program for preg7.

5 Secker-Walker RH, Solomon LJ, Flynn BS, Skelly JM, Mead PB. Reducing smoking in pregnancy and postpartum: Physician's advice supported by individual counseling. Prev Med 1998;27:422-30.

6 Kendrick J, Zahniser S, Miller N, Salas N, Stine J, Floyd R. Integrating smoking cessation into routine public prenatal care: the smoking cessation in pregnancy project. $A m^{f}$ Public Health 1995;85:217-22.

7 Lowe J, Balanda K, Clare G. Evaluation of antenatal smoking cessation programs for pregnant women. Aust $\mathrm{NZ} \mathcal{f}$ Public Health 1998;22:55-9.

8 Sexton M, Hebel R. A clinical trial of change in maternal smoking and its effect on birth weight. $\mathcal{F} A M A 1984$; 251:911-15.

9 Valbo A, Nylander G. Smoking cessation in pregnancy: intervention among heavy smokers. Acta Obstet Gynecol Scand 1994:73:215-19.

10 Walsh R, Redman S, Brinsmead M, Byrne J, Melmeth A. A smoking cessation program at a public antenatal clinic. $A m$ f Public Health 1997;87:1201-4.

11 Windsor R, Cutter G, Morris J, Reese Y, Manzella B, Bartlett $\mathrm{E}$. The effectiveness of smoking cessation methods for smokers in public health maternity clinics: a randomized trial. Am ₹ Public Health 1985;75:1389-92.

12 Windsor R, Boyd N, Orleans T. A meta-evaluation of smoking cessation intervention research among pregnant
women: improving the science and art. Health Educ Res 1998;13:419-38

13 Windsor RA, Lowe JB, Perkins LL, et al. Health education for pregnant smokers: its behavioral impact and cost benefit. Am ₹ Public Health 1993;83:201-6.

14 Windsor RA, Warner KE, Cutter GR. A cost-effectiveness analysis of self-help smoking cessation methods for analysis of self-help smoking cessation methods
pregnant women. Public Health Reports 1986;103:83-9.

15 Severson HH, Andrews JA, Lichtenstein E, Wall M, Akers L. Reducing maternal smoking and relapse: long-term L. Reducing maternal smoking and relapse: long-term 25:120-30.

16 DiClemente CC, Mullen PD, Pollak KI, Sockrider MM, Stotts AL. Intervention effects on pregnant quitters' partners' smoking. Proceedings of the annual meeting of the Society of Behavioral Medicine, New Orleans, 9-12 March, 1998.

17 Wakefield $\mathrm{M}$, Jones W. Effects of a smoking cessation program for pregnant women and their partners attending a public hospital antenatal clinic. Aust NZ 7 Public Health 1998;22:313-20.

18 Bergen AW, Caporosa N. Cigarette smoking. F Natl Cancer Inst 1999;91:1365-75.

19 Mullen PD, Pollak KI, Groff JY, DiClemente CC. Relations among psychosocial variables, addiction, and self-efficacy in lower and higher income pregnant smokers. Proceedings of the annual meeting of the Society for Research on Nicotine of the annual meeting of the Society for Research on Nico
and Tobacco, San Diego, California, 5-7 March, 1999.

20 Walsh RA, Redman S, Brindsmead MW, Fryer JL. Predictors of smoking in pregnancy and attitudes and
knowledge of risks of pregnant smokers. Drug Alcohol keview 1997;61:41-67.

21 Paarlberg KM, Vingerhoets JJM, Passchier J, Heinen AGJJ, Dekker GA, Van Geijn HP. Smoking status in pregnancy is associated with daily stressors and low well-being. Psychology Health 1999;14:87-96.

22 Waldron I. Patterns and causes of gender differences in smoking. Soc Sci Med 1991;32:989-1005.

23 Berman BA, Gritz ER. Women and smoking: current trends and issues for the 1990s. Fournal of Substance Abuse 1991, 3:221-38

24 National Center for Health Statistics/Center for Disease Control. Smoking during pregnancy, 1990-1996. National Vital Statistics Reports 1998;47:1-12.

25 Quinn VP, Mullen PD, Ershoff DH. Women who stop smoking spontaneously prior to prenatal care and predic-
tors of relapse before delivery. Addictive Behaviors 1991; 6:153-60.

26 Panjari M, Bell RJ, Astbury J, Bishop SM, Dalais F, Rice GE. Women who spontaneously quit smoking in early

27 Woodby LL, Windsor RA, Snyder SW, Kohler CL, DiClemente CC. Predictors of smoking cessation during pregnancy. Addiction 1999;94:283-92.
28 Appleton PL, Pharoah POD. Partner smoking behaviour change is associated with women's smoking reduction and cessation during pregnancy. British fournal of Health Psychology 1998;3:361-74.

29 McBride CM, Curry SJ, Grothaus LC, Nelson JC, Lando H, Pirie PL. Partner smoking status and pregnant
smoker's perceptions of support for and likelihood of smoking cessation. Health Psychology 1998;17:63-9.

30 Fingerhut LA, Kleinman JC, Kendrick JS. Smoking before, during and after pregnancy. Am f Public Health 1990; 80:541-4.

31 Mullen PD, Richardson MA, Quinn VP, Ershoff DH. Postpartum return to smoking: who is at risk and when. Am $\mathcal{F}$ Health Promotion 1997;11:323-30.

32 Ko M, Schulken ED. Factors related to smoking cessation and relapse among pregnant smokers. Am f Health Behavior 1998;22:83-9.

33 Stotts A, DiClemente CC, Carbonari JP, Mullen P. Pregnancy smoking cessation: a case of mistaken identity. Addictive Behaviors 1996;21:459-71.

34 DiClemente CC, Prochaska JO. Toward a comprehensive, ranstheoretical model of change: stages of change and addictive behaviors. In: Miller WR, Heather N, eds. Treating addictive behaviors, 2nd edn. New York: Plenum, 1998:3-23.

35 Stotts AL, DiClemente CC, Carbonari JP, Mullen PD. Postpartum return to smoking: staging a "suspended" behavior. Health Psychology In press.

36 McBride CM, Pirie PL. Postpartum smoking relapse. Addictive Behaviors 1990;15:165-8.

37 Lowe JB, Windsor R, Balanda KP, Woodby L. Smoking relapse prevention methods for pregnant women: a form tive evaluation. Am f Health Promotion 1997;11:244-6.

38 Benowitz NL. Nicotine replacement therapy during pregnancy. $\mathcal{F} A M A$ 1991;266:3174-7.

39 DiClemente CC, Mullen PD, Stotts AL. One to one: an intervention for resistant pregnant smokers. Proceedings of intervention for resistant pregnant smokers. Proceedings of Behavior Therapy, Washington, DC, 5-8, November, 1998.

40 Li C, Windsor R, Perkins L, Lowe J, Goldenberg R. The impact on birthweight and gestational age of cotinine validated smoking reduction during pregnancy. $\mathcal{F} A M A 1993$; 269:1519-24.

41 Windsor R, Li C, Boyd N, Hartmann K. The use of significant reduction rates to evaluate health education methods for pregnant smokers: a new harm reduction-behavioral indicator. Health Educ Behav 1999;26:648-62.

42 Hanna EZ, Faden VB, Dufour MC. The effects of substance use during gestation on birth outcome, infant and maternal health. Fournal of Substance Abuse 1997;9:111-26.

43 Mullen PD, DiClemente CC, Bartholomew LK. Theory and context and project PANDA: a program to help postpartum women stay off cigarettes. In: Bartholomew LK, evidence-based health promotion pragrams. Mountain View, California: Mayfield Publishers, 2000: 453-77.

44 McBride CM, Curry SJ, Lando HA, Pirie PL, Grothaus LC, Nelson JC. Prevention of relapse in women who quit smoking during pregnancy. Am f Public Health 1999; 89:706-11.

45 US Department of Health and Human Services: The Public Health Service expert panel on the Content of prenatal care. Caring for our future: the content of prenatal care. Washington, DC: US Government Printing Office, 1989.

46 DiClemente CC. Motivation for change: implications for substance abuse treatment. Psychological Science 1999; 10:209-13.

47 Ershoff DH, Quinn VP, Boyd NR, Stern J, Gregory M, Wirtshafter D. The Kaiser Permanente prenatal smoking cessation trial: when more isn't better, what is enough? Am f Prev Med 1999;17:161-8.

48 Solomon LJ, Secker-Walker RH, Skelly JM, Flynn BS. Stages of change in smoking during pregnancy in low-income women. F Behav Med 1996;19:333-44.

49 Mullen PD. Maternal smoking during pregnancy and evidence based intervention to promote cessation. Primary Care 1999;26:577-89.

50 Adams EK, Young TL. Costs of smoking: a focus on maternal, childhood, and other short-run costs. Med Care Res Rev 1999;56:3-29. 